\title{
Accurate Measurement of Both Main Components of Heart Rate Variability Can Be Applied Routinely Clinically
}

\author{
Gary L Murray* \\ The Heart and Vascular Institute, USA
}

\section{SHORT COMMUNICATION}

One century ago, the critical role of the Autonomic Nervous System (ANS) in health and disease was prophesized. In 1990, Heart Rate Variability (HRV = Sympathetic-tone [S] + Parasympathetictone [P]) was $1^{\text {st }}$ used in clinical cardiology. Emphasizing the reduction in HRV was associated with a poor prognosis in all major cardiovascular illness. In 2000, HRV was included in Sudden Cardiac Death (SCD) risk stratification. There are over 350,000 SCDs/yr. in the USA. High S and critically low $\mathrm{P}$ are associated with increased mortality, life-threatening ventricular arrhythmias, congestive heart failure (CHF), and acute coronary syndromes (ACSs). Two common autonomic dysfunctions are Sympathetic Withdrawal (SW, an abnormal alpha-adrenergic response to head-up postural change underlying Orthostatic dysfunction) and Parasympathetic Excess (PE, an abnormal cholinergic response to stress, amplifying the stress response, as in difficult to manage blood pressure (BP), blood glucose, hormone levels, or Anxiety syndromes). Both SW and PE contribute significantly to the worldwide pandemic of uncontrolled Hypertension (HTN). Both result in poor brain perfusion leading to an increase in resting BP as compensatory, an increase in resting BP that is largely resistant to anti-hypertensives. On average, only $35 \%$ of Hypertensives are well managed, increasing the mortality risk of 1.5 billion people with HTN [1-9]. And yet, ANS testing is very rarely used in patient management, probably mainly due to difficulty in directly measuring P. All non-invasive ANS measurements only measure total autonomic activity, resulting in assumptions and approximations of the independent contributions of $\mathrm{S}$ and $\mathrm{P}$ to total HRV. Since HRV $=\mathrm{S}+\mathrm{P}$, both $\mathrm{S}$ and $\mathrm{P}$ must be accurately identified.

However, there is one exception. A technologic breakthrough was developed, validated, and verified by the $1^{\text {st }}$ joint Bio-Medical
Engineering program group from Massachusetts Institute of Technology and Harvard [10-14], and is now available for userfriendly routine clinical use. It is $\mathrm{P} \& S$ Monitoring. The breakthrough quantifies the independent contributions of $\mathrm{S}$ and $\mathrm{P}$ to total HRV through two simultaneous measurements: (1) ECG monitoring which establishes total HRV (Low Frequency area [0.04-0.15 Hz] under the HR time-frequency spectral curve), simultaneously with (2) Impedance Plethysmography which independently quantitates P (a $0.12 \mathrm{~Hz}$-wide window area under the HRV spectral curve centered on the modal peak of the time-frequency Respiratory Activity (RA) spectral curve; HRV due to RA is solely P-dependent). Therefore, $\mathrm{S}=\mathrm{HRV}$ - $\mathrm{P}$; where $\mathrm{P}$ is no longer assumed to be the area under the curve between $0.15-0.40 \mathrm{~Hz}$, but now is quantitatively measured as the Respiratory Frequency area. The curves are analyzed using continuous wavelet transforms rather than the frequency-only fast Fourier transforms. The latter, although accurate for stationary signals, compromises time and frequency resolution due to the fixed length windows used in analysis. The value of routinely using this new technology is illustrated by our following studies.

\section{HYPERTENSION}

Comparing ANS-assisted to JNC 8 treatment, $74 \%$ of the ANSassisted patients reached JNC 8 treatment control goals vs. $30.4 \%$ of the JNC 8 patients within 9 mo. ( $p<0.001$ for both systolic and diastolic BP) on 2.3 vs. 3 prescription anti-hypertensives. Final S was lower and $\mathrm{P}$ higher (both $\mathrm{p}<0.001$ ) in the ANS-assisted group [9].
Quick Response Code:

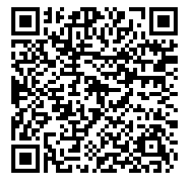

Address for correspondence: Gary L Murray MD, FACC, FICA, Director, Clinical Research, The Heart and Vascular Institute, USA

Received: January 25, 2020

Published: February 05, 2020

How to cite this article: Gary L Murray. Accurate Measurement of Both Main Components of Heart Rate Variability Can Be Applied Routinely Clinically. 2020 - 2(1) OAJBS.ID.000138. DOI: $10.38125 / \mathrm{OAJBS} .000138$ 


\section{AUTONOMIC BALANCE IN CHF}

Beta blockers are the cornerstone of CHF pharmacologic therapy. Of concern, 59\% of 54 patients had either high ( $>3$ ) Sympathovagal Balance $(\mathrm{SB}$, a resting measure, $=\mathrm{S} / \mathrm{P}$ ), Cardiovascular Autonomic Neuropathy (CAN, a resting measure, $<0.1 \mathrm{bpm}^{2}$ ) or both, despite guideline-mediated treatment. Off-label Ranolazine corrected most of these treatment failures [15]. In another study of 109 patients [16], in addition to improving SB and relieving CAN, Ranolazine (titrated with ANS guided therapy) independently improved left ventricular ejection fraction in $70 \%$ of patients; on average 11 units, $\mathrm{p}=0.018$ (vs. no change in the usual treatment group). In this other study, by titrating Ranolazine to normalize SB and thereby helping to relieve CAN, the outcome was to reduce cardiac deaths, acute CHF, and malignant ventricular arrhythmias in the ANS assisted therapy patients as compared with the usual treatment group. Given the critical role of beta blockade in CHF, it seems essential to measure S on therapy and adjust accordantly. Presently, this is never done.

\section{RISK STRATIFICATION IN PATIENTS AT RISK FOR CARDIOVASCULAR DISEASE OR WITH ESTABLISHED DISEASE}

In 483 patients (127 with risk factors, 224 with coronary disease, 132 with chronic CHF) followed for a mean of 4.92 yrs., $\mathrm{SB}>2.5$ outperformed myocardial perfusion imaging (MPI) and echocardiography (2DE) $(\mathrm{p}=0.001)$ with a sensitivity of 0.59 , $\mathrm{OR}=7.03$ (CI: 4.59-10.78), specificity of $0.83, \mathrm{PPV}=0.64$, and $\mathrm{NPV}=0.80$ for predicting cardiac death, ACS, acute CHF, and malignant ventricular arrhythmias [17]. Since only $31 \%$ of the patients had $\mathrm{SB}>2.5$, perhaps they require MPI and/or 2DE, and the other $69 \%$ of stable patients can avoid expensive imaging procedures.

\section{NEUROGENIC ORTHOSTATIC HYPOTENSION $(\mathrm{NOH})$}

Ten to $30 \%$ of the elderly experience Orthostatic Hypotension $(\mathrm{OH})$, resulting in profound morbidity and increased mortality. Currently, routine management consists of compression stockings, vasopressors, mineralocorticoids, fluids, and more recently Droxydopa. Low $\mathrm{S}$ in response to standing (SW) is a very common cause of $\mathrm{NOH}$, not always addressed by stockings, vasopressors, mineralocorticoids, fluids; and Droxydopa is expensive. In 109 patients with low-S Orthostatic Intolerance, the OTC antioxidant (r-) Alpha Lipoic Acid (ALA) had a 66\% response rate via increasing $\mathrm{S}$ and avoiding the inconvenience, expense, and side effects of current therapies, such as supine/sitting HTN [18].

\section{CONCLUSION}

In the $2^{\text {nd }}$ century AD, Wang Shu Ho stated, "If the heartbeat gets as regular as the knocking of the woodpecker or the dripping of the rain on the roof, the patient will die within 4 days". And yet, analysis of HRV remains unused. What a shame; science has made it easy: with P\&S Monitoring, now HRV DOES $=\mathrm{S}+\mathrm{P}$.

\section{REFERENCES}

1. Vinik A, Ziegler D (2007) Diabetic cardiovascular autonomic neuropathy. Circulation 115(3): 387-397.

2. Tomaselli G, Zipes D (2004) What causes sudden death in heart failure? Circ Res 95: 754-763.

3. Maser R, Mitchel B, Vinik A, Freeman R (2003) The association between cardiovascular autonomic neuropathy and mortality in individuals with diabetes: a meta-analysis. Diabetes Care 26(6): 1895-1901.

4. Watanabe J, Shinokaki T, Shiba N, Fukahori K, Koseki Y, et al. (2006) Accumulation of risk markers predicts the incidence of sudden death in patients with heart disease. Eur J Heart Fail 14(8): 237-243.

5. Curtis B, O Keefe J (2002) Autonomic tone as a risk factor: the dangers of chronic fight or flight. Mayo Clinic Proc 72(1): 45-54

6. McCance A, Thompson P, Forfar J (2006) Increased cardiac sympathetic nervous activity in patients with coronary heart disease. Eur j Heart Fail 14(6): 751-757.

7. Manfrini O, Morgacni C, Pizzi C, Fontana F, Burgiardini R (2004) Changes in autonomic nervous activity: spontaneous versus balloon-induced myocardial ischemia. Eur Heart J 25(3): 1502-1508.

8. Akuttsu Y, Kaneko K, Kodama Y, Sayama J, Shinozuka A, et al. (2007) Significance of cardiac sympathetic nervous system abnormality for predicting vascular events in patients with coronary artery disease. Eur J Nucl Mol Imaging 7(17): 742-749.

9. Murray G. The freasibility of blood pressure control with autonomicassisted hypertension therapy versus JNC 8 therapy.

10. Aysin B, Colombo J, Asyn E (2007) Comparison of HRV analysis methods during orthostatic challenge: HRV with respiration or without? $29^{\text {th }}$ Int Conf IEEE EMBS 2007, Lyon, France.

11. Akselrod S, Gordon D, Ubel F, Shannon D, Berger A, et al. (1981) Power spectrum analysis of heart rate fluctuation: a quantitative probe of beatto-beat cardiovascular control. Science 213(4504): 220-222.

12. Akselrod S, Gordon D, Madwed J, Snidman N, Shannon D, et. al. (1985) Hemodynamic regulation: Investigation by spectral analysis. Am J Physiol 249: H867-H875.

13. Akselrod S, Eliash S, Oz O, Cohen S (1987) Hemodynamic regulation in SNR: investigation by spectral analysis. Am J Physiol 253: H176-H183.

14. Akselrod S (1989) Spectral analysis of fluctuations in cardiovascular parameters: a quantitative tool for the investigation of autonomic control. Trends Pharmacol Sci 9(1): 6-9.

15. Murray G, Colombo J (2014) Ranolazine improves autonomic balance in heart failure when added to guideline-driven therapy. Heart International 9(2): 1-7.

16. Murray G, Colombo J (2014) Ranolazine preserves and improves left ventricular ejection fraction and autonomic measures when added to guideline-driven therapy in chronic heart failure. Heart Int 9(2): 66-73.

17. Murray G, Colombo J (2019) Routine measurements of cardiac parasympathetic and sympathetic nervous systems assists in primary and secondary risk stratification and management of cardiovascular clinic patients. 3(1): 27-33

18. Murray G, Colombo J (2019) (r)Alpha Lipoic Acid is a safe, effective pharmacologic therapy of chronic orthostatic hypotension associated with low sympathetic tone. J Int Coll Angiol 28: 188-193. 